\title{
Recycling of cell surface membrane proteins from yeast endosomes is regulated by ubiquitinated Ist 1
}

\author{
Kamilla ME. Laidlaw ${ }^{1}$, Grant Calder ${ }^{2}$, Chris MacDonald ${ }^{1 *}$ \\ 1 York Biomedical Research Institute and Department of Biology, University of York, York, UK \\ ${ }^{2}$ Imaging and Cytometry Laboratory, Bioscience Technology Facility, Department of Biology, University of York, UK \\ *Correspondence: Email: chris.macdonald@york.ac.uk Tel: +44 (0) 1904328609
}

\begin{abstract}
Trafficking of cell surface membrane proteins to and from the plasma membrane impinges on myriad biological processes and ensures correct cellular function. Upon internalisation, most surface proteins are either sent to the lysosome for degradation or recycled back to the plasma membrane. Although these endosomal trafficking pathways control surface protein activity, the precise regulatory features and division of labour between interconnected pathways is poorly defined. Furthermore, how well endosomal trafficking mechanisms are conserved across eukaryotes is unclear. In yeast, we show cargo recycling back to the plasma membrane occurs through distinct pathways. In addition to the established retrograde recycling pathways via the late Golgi, which are used by synaptobrevins and driven by cargo ubiquitination, we find nutrient transporter recycling bypasses the Golgi in a pathway driven by cargo deubiquitination. Nutrient transporters rapidly internalise to, and recycle from, endosomes marked by Vps 4 and the ESCRT-III associated factor Ist1. This endosome population serve as both 'early' and 'recycling' endosomes, implying these features of endosomal organisation are evolutionarily conserved. Ist1 has previously been implicated in recycling in yeast and other eukaryotes. We reveal Ist1 ubiquitination regulates its endosomal recruitment and is required for cargo recycling. Additionally, the ubiquitin-binding adaptor Npl4 and the essential ATPase Cdc48 regulate endosomal Ist1 and are required for cargo recycling, suggesting mechanistic features of recycling from endosomes to the plasma membrane are also conserved.
\end{abstract}

\section{INTRODUCTION}

The plasma membrane (PM) hosts a variety of functionally diverse proteins that are regulated by intracellular trafficking pathways. Surface proteins can be downregulated through endocytosis and trafficking to the lysosome (vacuole in yeast), for example specific downregulation of receptors / transporters recognising ligand / substrate (Davis et al., 1993; Séron et al., 1999) or more general cargo endocytosis following stress (Laidlaw et al., 2020; Müller et al., 2015).(Laidlaw et al., 2020; MacGurn et al., 2011; Müller et al., 2015) Endocytosed surface cargoes that retain a ubiquitination signal are targeted through the multivesicular body (MVB) pathway, packaged into intraluminal vesicles (ILVs) and then delivered to the vacuole for degradation (Katzmann et al., 2001; Urbanowski and Piper, 2001). Ubiquitinated cargoes are recognised by the Endosomal Sorting Complex Required for Transport (ESCRT) complexes, with ESCRT-0 and ESCRT-I abundant in ubiquitin binding domains(Shields et al., 2009). ESCRT-III subunits are recruited to the endosomal/MVB membrane, where they polymerise and drive formation of vesicles budding into the lumen of the endosome (Hanson et al., 2008; Saksena et al., 2009; Wollert et al., 2009). Filaments created through polymerisation of Snf7, modulated by other ESCRTs and the Vps4 ATPase drive membrane deformation (Adell et al., 2017; Maity et al., 2019; Pfitzner et al., 2020; Shestakova et al., 2010; Tang et al., 2015). Nutrient transporters in yeast are useful reporters for endosomal trafficking, such as the amino acid transporters Mup1 and Fur4, as their ubiquitination and ESCRT-dependent trafficking to the vacuole can be triggered by addition of substrate (Hein et al., 1995; Isnard et al., 1996; Keener and Babst, 2013). The E3-ligase Rsp5 and its substrate specific adaptors is largely responsible for cargo ubiquitination and vacuolar sorting events (Sardana and Emr, 2021). Surface activity of internalised PM proteins is also regulated by recycling routes back to the PM (MacDonald and Piper, 2016). Recycling in yeast appears less complex than mammalian cells but the division of labour between certain pathways and even organisation of the endosomal system, is not fully understood (Day et al., 2018; Laidlaw and MacDonald, 2018; Ma and Burd, 2019). Retrograde recycling of yeast synaptobrevin orthologues, Snc1 and Snc2 (Protopopov et al., 1993), via the trans-Golgi network (TGN) has been extensively studied. Localisation of Snc1 is polarised with concentration to the bud-tips of emerging daughter cells and the shmoo protrusions induced upon response to mating factor; this polarisation relies on post-endocytic recycling (Valdez-Taubas and Pelham, 2003). Snc1 recycles via the TGN through multiple pathways involving different machinery, such as retromer, Snx4-Atg20 (Hettema et al., 2003; Lewis et al., 2000; Ma et al., 2017) and other 
factors, such as phospholipid flippases, Rcy1, and Ypt31/32 (Chen et al., 2005; Furuta et al., 2007; Galan et al., 2001; Hanamatsu et al., 2014; Hua et al., 2002). Retrograde recycling of Snc1 also requires its ubiquitination to facilitate interaction with endosomally localised COPI (Xu et al., 2017). However, deubiquitination of other cargoes, like nutrient transporters that are typically sorted to the vacuole upon ubiquitination, appears to have the opposite effect. Directing catalytic activity of deubiquitinating enzymes (DUb) to cargo, either directly (MacDonald et al., 2015; Stringer and Piper, 2011) or indirectly via Rsp5 and ESCRT proteins (MacDonald et al., 2012a, 2012b; Stringer and Piper, 2011), antagonises cargo degradation and triggers recycling by default. To characterise this pathway, a genetic screen using a DUb fused version of the receptor Ste3 (Ste3-GFPDUb) was performed to reveal 89 factors that are required for this recycling (MacDonald and Piper, 2017), most of which were validated with additional assays for Tat2 (Johnson et al., 2010) and FM4-64 (Wiederkehr et al., 2000) recycling. Null mutants of retrograde machinery like retromer, Snx4/41/42 and Ere1/2 (Hettema et al., 2003; Seaman et al., 1998; Shi et al., 2011) were not identified by the screen and recycle FM4-64 at similar rates to wild-type cells. Furthermore, cells harbouring a mutant allele of SEC7 with abrogated trafficking through the Golgi also exhibit efficient DUb-triggered recycling. These data suggest the recycling of DUb-fused cargoes is predominantly distinct from retrograde recycling of Snc1, but there is likely overlap between routes as some endosomal machinery that Snc1 relies on was shown to be required for Ste3-GFP-DUb recycling (Rcy1, Cdc50Drs2, Ypt31/32).

Ist1 was also confirmed to be required for the recycling pathway triggered by deubiquitination. Ist1 shares structural homology with other ESCRT-III subunits and contributes to the efficiency of MVB sorting (Buono et al., 2016; Dimaano et al., 2008; Frankel et al., 2017; Pfitzner et al., 2020; Rue et al., 2008; Xiao et al., 2009). Ist1 interacts with other ESCRT-III subunits and Vps4, the AAA-ATPase required for MVB sorting and disassembly of ESCRT polymers (Azmi et al., 2006; Babst et al., 1997, 1998, 2002; Nickerson et al., 2006). Ist 1 regulation of Vps4 is complex, even in vitro Ist1 can stimulate and inhibit Vps4 activity and control depends on other ESCRT-III subunits (Tan et al., 2015). Therefore, despite great strides in our understanding of ILV formation by ESCRT-III filaments and Vps4 (Adell et al., 2017; Han et al., 2017; Maity et al., 2019; Pfitzner et al., 2020) our understanding of the role(s) of Ist1 in the endosomal assemblage of ESCRTs in vivo is incomplete. Furthermore, high levels of Ist1 inhibit MVB sorting and diverse cargoes are sorted more efficiently to the vacuole in ist1 $\Delta$ cells (Jones et al., 2012). In addition to the negative regulation of Vps4, it may be that Ist1 also promotes an opposite acting recycling pathway to the PM. In support of this idea, in vivo and in vitro studies show that unlike ESCRT-III polymers, which drive luminal vesicle formation, Ist1 polymerisation exerts the opposite effect on endosomal membranes to generate tubulation of cytosolic protrusions (McCullough et al., 2015). Physiologically this can be best rationalised by Ist 1 promoting the recycling pathway by creation / fission of recycling tubules that return material back to the PM in collaboration with the ATPase spastin (Allison et al., 2013, 2017).

In this study we present evidence that Snc1/2 mainly follow a trafficking route to the TGN that is distinct from the pathway used by nutrient transporters from methionine (Mup1) and uracil (Fur4). Upon addition of substrate, nutrient transporters rapidly internalise to compartments marked by Vps4 and Ist1, followed by delivery to the vacuole. However, upon removal of the substrate-induced ubiquitin-degradation signal, Mup1 recycles directly back to the PM from this endosome population. This recycling pathway driven by deubiquitination relies on the ESCRT-III associated protein Ist1, and we provide initial evidence that Ist1 ubiquitination and Npl4-Cdc48 is required for proper endosomal regulation of Ist1 mediated cargo recycling.

\section{RESULTS}

\section{Differential cargo recycling routes in yeast}

The V-SNARE Snc1 is a well-established retrograde cargo that internalises and recycles via the TGN back to the plasma membrane (PM) through multiple pathways (Best et al., 2020; Lewis et al., 2000). Snc1 ubiquitination is required for recycling (Xu et al., 2017) and the fusion of a deubiquitinating enzyme (DUb) is sufficient to block recycling of GFP-tagged Snc1 and the paralogue Snc2 (Figure 1A). In contrast, substrate induced ubiquitination of the Mup1 (methionine) or Fur4 (uracil) permeases does not promote recycling and instead drives endocytosis and vacuolar degradation (Hein et al., 1995; Keener and Babst, 2013; Lin et al., 2008; Menant et al., 2006). The fusion of either Mup1 or Fur4 to a DUb domain antagonises this vacuolar sorting (Stringer and Piper, 2011), resulting in PM localisation of transporters, even in the presence of substrate (Figure 1B). Retrograde recycling of Snc1/2 via the TGN is required for its polarised PM distribution (Hettema et al., 2003; Lewis et al., 2000; Ma et al., 2017; Valdez-Taubas and Pelham, 2003), with GFP tagged Snc1/2 concentrated in budding daughter cells (Figure 1C-1E). Unlike Snc1/2, fluorescently tagged Mup1 and Fur4 concentrate in the mother cell during budding (Figures S1A-E), with polarisation maintained through the cell cycle for Fur4 but being chiefly in small cells $(<2.5 \mu \mathrm{m}$ diameter) for Mup1. As expected, intracellular signal from retrograde cargoes Snc1/2 colocalises with the TGN marker Sec7-mCherry, with very little overlap with 
the endosomal marker Vps4-mCherry (Figure 1F). Furthermore, DUb-GFP-Snc1/2 fusions, which do not recycle efficiently, accumulate in Sec7-marked TGN compartments (Figure 1G). The majority of intracellular signal is recycling Snc1, and not first-pass molecules transiting the Golgi, as a mutant version of Snc1 (Snc1 ${ }^{\mathrm{PM}}$ ) with defective internalization (Lewis et al., 2000) exhibits very little intracellular signal, which maintains colocalisation with Sec7-mCherry (Figure 1H). In contrast, intracellular Mup1-GFP, triggered by substrate or nutrient starvation (MacDonald et al., 2012b, 2015) primarily internalises to Vps4-mCherry, and not TGN, compartments (Figure 1I). Similarly, Fur4-mNG that has significant intracellular signal irrespective of substrate presence colocalises with Vps4 endosomes (Figure 1J). Although cargo-specific trafficking regulation might explain mother-daughter differences, taken with the opposing effects of enforced deubiquitination and differences in intracellular localisation, we propose that PM recycling of nutrient transporters follows a distinct route than Snc1/2 (Figure 1K).

\section{Nutrient transporters recycle from early endosomes}

Although enforced deubiquitination of nutrient transporters promotes PM recycling, observing recycling of unmodified nutrient transporters is hampered by their proclivity for vacuolar sorting (MacDonald et al., 2012a, 2012b, 2015; Stringer and Piper, 2011). To overcome this, we optimised microfluidic exchange with continuous imaging to perform a substrate pulse, followed by wash and substrate-free chase to allow internalised cargo to recycle naturally (Figure 2A). Mup1-GFP was used for these experiments as it exhibits steady state localisation at the PM, and we found high $(40 \mu \mathrm{g} / \mathrm{ml})$ and low $(2 \mu \mathrm{g} / \mathrm{ml})$ levels of methionine triggered accumulation of intracellular puncta, with high doses giving brighter, more obvious puncta (Figure 2B). The pulse-chase protocol resulted in accumulation of intracellular Mup1-GFP for 0-15 minutes followed by clearance of most signal after an additional 15 minutes, regardless of methionine pulse concentration. To avoid concerns about photobleaching or substrate induced degradation, biochemical analysis was used to show that methionine pulse periods of 1 or 5 minutes, followed by substrate free chase up to 60 minutes resulted in no increase in vacuolar delivery of this internalised material (Figure 2C-2D). All subsequent substrate induced experiments were performed with $<1$ minute methionine pulses. To confirm intracellular nutrient transporter signal emanated from the surface, we optimised photoconversion of surface localised Mup1-mEos and Fur4-mEos (Figure 2E-2F, S2A-S2C). Coupling cargo photoconversion to microfluidic induced recycling and time-lapse microscopy, revealed signal of intracellular Mup1 from the PM, which subsequently dissipates with similar kinetics to Mup1GFP (Figure 2G, Movie S1). We then followed the trafficking itinerary of internalised and recycled Mup1-GFP using 4D confocal Airyscan microscopy optimised for rapid acquisitions. Methionine induced internalisation tracked at $4-10$ second intervals show substantial intracellular accumulations that colocalise with Vps4mCherry, and not Sec7-mCherry within the first few minutes (Figure 3A, Movie S2-S3). Additionally, Mup1GFP recycling experiments were imaged over longer periods to show Mup1 primarily traverses Vps4 endosomes, bypassing Sec7-mCherry compartments (Figure 3B-3C, Movie S4-S5). We note that Mup1-GFP colocalises to a small but significant degree with a population of Sec7-mCherry adjacent to the PM (Figure S3A-S3C), which might be explained by rapid trafficking to TGN compartments in the vicinity of the PM or an artifact from the deconvolved micrographs.

\section{Ist1 is required for cargo recycling}

Vps4, the ATPase involved in ESCRT-mediated ILV formation, marks a large and relatively static endosome reminiscent of Vps8 compartments (Day et al., 2018) in addition to a population of peripheral mobile compartments (Adell et al., 2017). The ESCRT-associated factor Ist1 colocalises with Vps4 in both populations, in addition to potentially distinct endosomes (Figure 3D, Movie S6). As Ist1 was the only recycling factor from a blind genetic screen (MacDonald and Piper, 2017) that also interacts with Vps4 (Figure 3E), and its orthologues have been implicated in recycling (Allison et al., 2013; Frankel et al., 2017; McCullough et al., 2015), we hypothesised Ist 1 might functionally define this population of recycling endosomes. We integrated the Ste3-GFP-DUb recycling reporter to confirm recycling defects of ist1 $\Delta$ and $r c y 1 \Delta$ cells, in addition to quantifying their capacity for recycling Tat2 and FM4-64 (Figure 3F-3H). As Ist1 has been implicated in polymerisation and creation of cytosolic recycling tubules (Allison et al., 2013, 2017; McCullough et al., 2015), we reasoned this function could be conserved in yeast (Figure 4A). Vps4 is an obvious candidate AAA-ATPase for Ist1 depolymerisation. However, vps $4 \Delta$ cells exhibit only a marginal defect in FM4-64 recycling (MacDonald and Piper, 2017) and a mutant of Ist1 (Ist $1^{\Delta \mathrm{MIM}}$ ) with significantly diminished Vps4-binding (Dimaano et al., 2008; Tan et al., 2015) rescues efficient FM4-64 recycling of ist1 $\Delta$ cells (Figure 4B). Other mutants that affect Vps4 activity (Tan et al., 2015) also recycle FM4-64 efficiently (Figure S4A). Furthermore, vps4 $\Delta$ cells or wildtype cells expressing a dominant negative E233Q mutation (Babst et al., 1998) efficiently recycle Ste3-GFPDUb (Figure 4C). 


\section{Npl4-Cdc48 regulates Ist1-recycling}

Screening for recycling machinery did not identify any other ATPase candidates but did implicate Npl4, an adaptor of the essential Cdc48 ATPase, as required for recycling (Figure S4B). We demonstrate that both Npl4 (Figure 4D-4E) and Cdc48 (Figure 4F-4G) are required to efficiently recycle Ste3-GFP-DUb and FM4-64 back to the PM. This led to our speculative model that the role of Cdc48-Npl4 in recycling was mediated via Ist1, and that Ist1 ubiquitination would allow Cdc48 recruitment via the well-established Npl4-ubiquitin binding motif (Wang et al., 2003). Although Ist1 is known to be turned over by the proteasome (Jones et al., 2012), it has not been formally demonstrated to be ubiquitinated, likely owning to the abundance of Lys / Arg residues (50/298 amino acids) that promote extensive tryptic digestion and prohibit identification of ubiquitin modifications by mass-spectrometry. We therefore performed a denatured ubiquitome purification (MacDonald et al., 2017, 2020; Xu et al., 2017) to reveal that ubiquitinated species of Ist1 can be observed (Figure 4H). Our model would predict that ubiquitination of Ist 1 is required for recycling, promoting recruitment of Cdc48 via Npl4 (Figure 4I). In support of this, Ist1-HA supports recycling of ist1 $\Delta$ cells to wild-type levels, but a mutant resistant to ubiquitination (Ist $1^{\mathrm{KR}}$-HA) cannot (Figure $4 \mathrm{~J}-4 \mathrm{~K}$ ). In support of a model for Ubiquitin $>\mathrm{Npl} 4>\mathrm{Cdc} 48$ regulating endosomal cycling of Ist 1 , we find elevated endosomal retention of Ist $1^{K R}$-GFP, which cannot be ubiquitinated (Figure 4L-4M). Furthermore, elevated endosomal levels of Ist1-GFP is observed in $n p / 4 \Delta$ cells and mutants with reduced Cdc48 ATPase activity. Combining the lysineless version of Ist $1^{\mathrm{KR}}$-GFP with Npl4 / Cdc48 mutants results in its mislocalisation to the nucleus (Figure S4C), which precluded assessment of endosomal recruitment but further alludes to a biological connection between ubiquitnated Ist1 and Npl4-Cdc48. Although perturbing Ist1-GFP ubiquitination by fusion with a DUb results in colocalization with the large, static prevacuolar Vps4 population, there is an observable separation of Vps4 and Ist1 in the small mobile population (Figure 4N). Importantly, these compartments are also distinct from the TGN (Figure S4D). This suggests that although Vps4 and Ist1 occupy the same compartments in wild-type conditions, that distinct trafficking intermediates can emanate from these endosomes.

\section{SUMMARY}

We show that retrograde recycling of Snc1/Snc2 is distinct from the recycling of the Mup1/Fur4 nutrient transporters. Snc1/2 mainly internalise to Sec7-marked TGN compartments, exhibit polarised distribution in daughter cells and rely on cargo ubiquitination (Figure 1). In contrast, Mup1/Fur4 internalise to Vps4-marked endosome compartments, localise predominantly to mother cells during budding, and deubiquitination triggers their recycling. Ubiquitination of nutrient transporters like Mup1 in response to substrate (Schothorst et al., 2013) can be counteracted by substrate removal, which triggers cargo recycling from Vps4 compartments back to the PM (Figures 2 \& 3). Given Mup1 internalises to Vps4 endosomes on the scale of seconds to minutes, these compartments can be considered yeast early endosomes. Furthermore, our interpretation of the imaging and biochemical data presented is internalised Mup1 recycles back to the surface directly from these endosome compartments, allowing them to be considered as yeast recycling endosomes. Although endosomal recycling of cargo in mammalian cells is complex (Goldenring, 2015; Grant and Donaldson, 2009; O'Sullivan and Lindsay, 2020), these observations support the notion that recycling features are evolutionarily conserved and can be elucidated using yeast. To this end, we reveal mechanisms of action of the yeast orthologue of IST1, which regulates endosomal recycling in animal cells (Allison et al., 2013, 2017; McCullough et al., 2015). We demonstrate that yeast Ist 1 is ubiquitinated, which is required for proper endosomal recruitment and recycling capacity (Figures 4). Recently, regulation of the IST1 partner CHMP1B has also been shown through ubiquitination (Crespo-Yàñez et al., 2018), alluding to a general posttranslational means to control ESCRTpolymerisation in other modes of actions. In addition to ubiquitination of Ist1, we show the ubiquitin-adaptor $\mathrm{Npl} 4$ and its ATPase partner Cdc48 (p97 / VCP) regulate endosomal recruitment of Ist1 and are required for efficient cargo recycling to the PM. It is tempting to speculate that the role of Ist1 in yeast recycling depends on its polymerisation, analogous to its mammalian counterpart, and that the Ubiquitin $>\mathrm{Npl} 4>\mathrm{Cdc} 48$ pathway uncovered herein serves to depolymerise and maintain Ist1 cycling. 
A

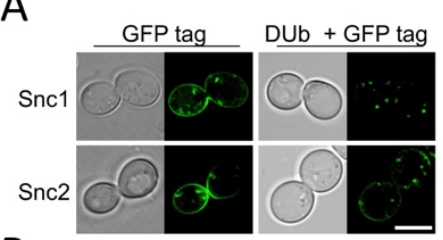

B

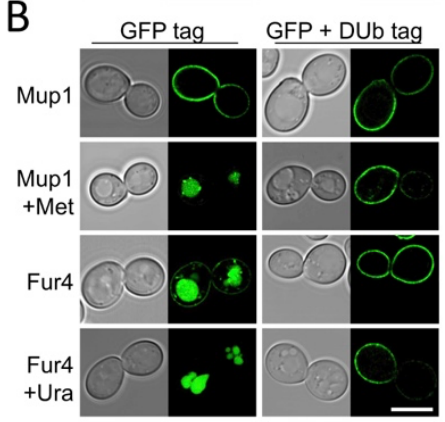

$\mathrm{F}$

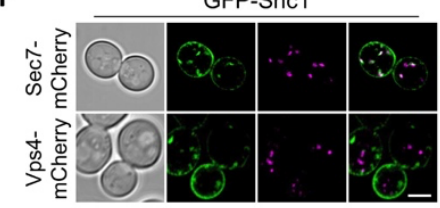

GFP-Snc2

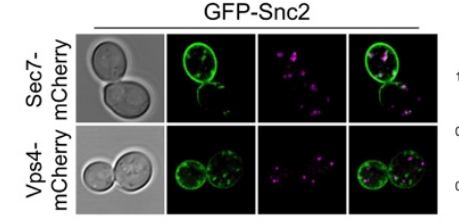

G
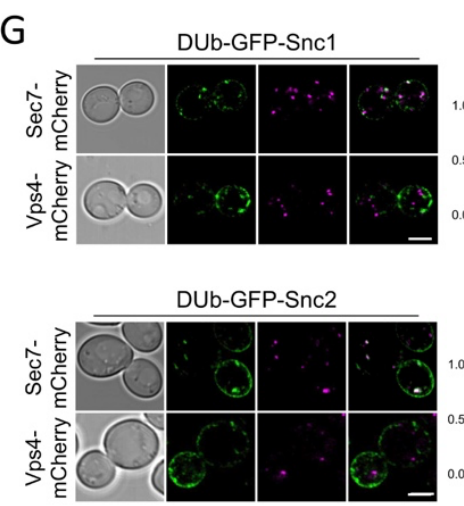

$\mathrm{H}$

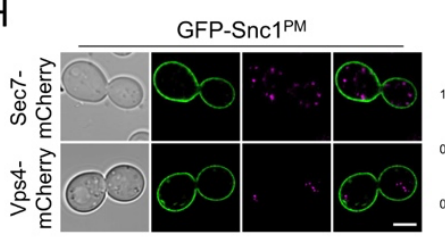

C

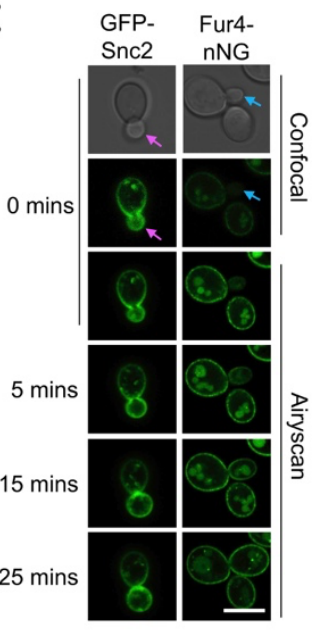

D

$\mathrm{E}$
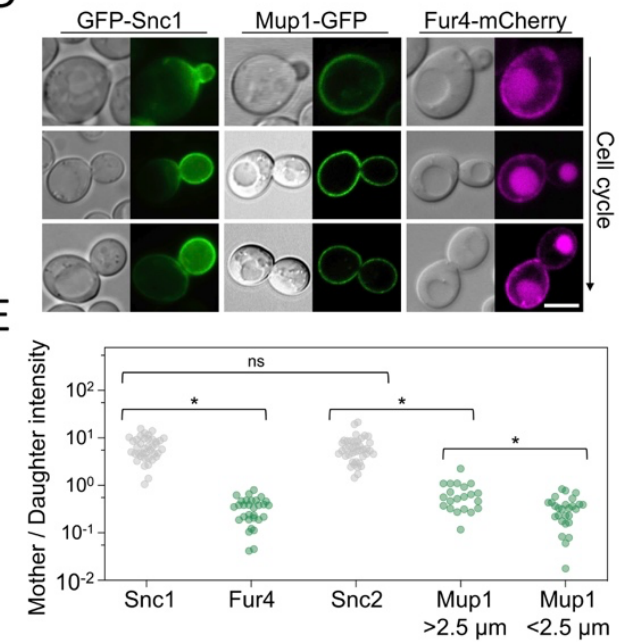

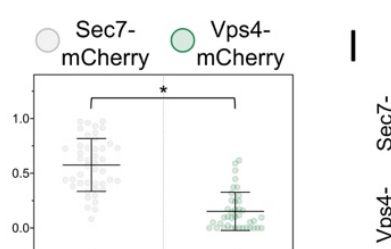

Sec7- Vps4-

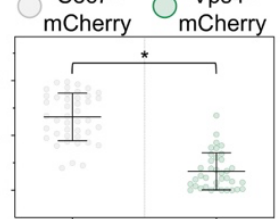
Sec7-
mCherry mps4-
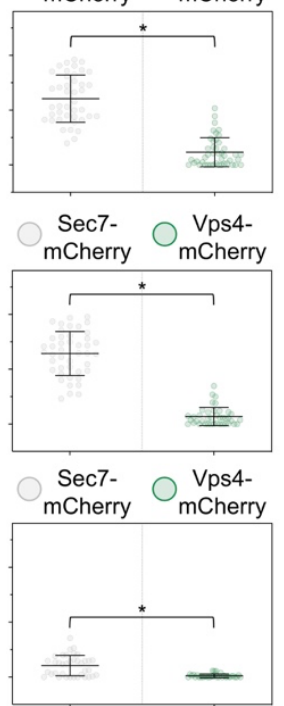
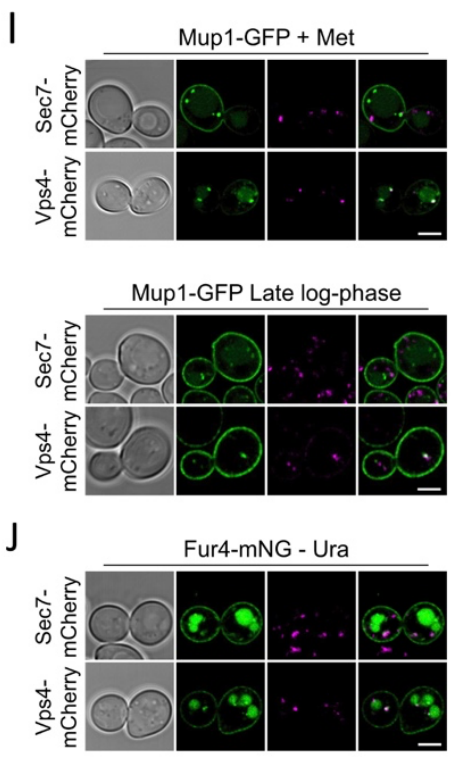

Fur4-mNG + Ura

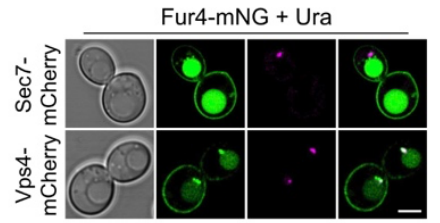

$\mathrm{K}$
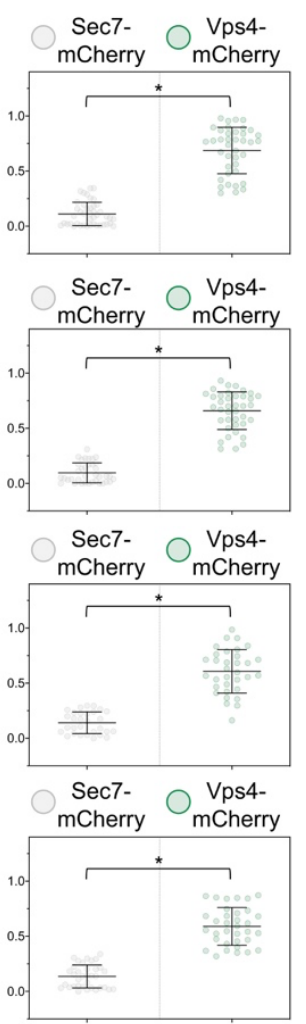

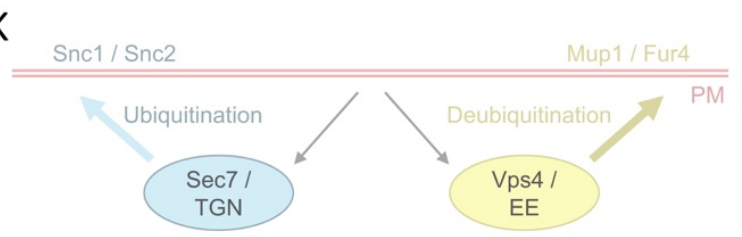

\section{Figure 1: Differential trafficking features of recycling cargoes}

A) Wild-type cells expressing Snc1 and Snc2 tagged with either GFP or a fusion of GFP with the catalytic domain of deubiquitinating enzyme UL36 (DUb + GFP) expressed from the CUP1 promoter were imaged by Airyscan microscopy. B) Mup1 and Fur4 expressed from their endogenous promoters and fused to C-terminal GFP or GFP-DUb tags were imaged by Airyscan microscopy. Where indicated, $20 \mu \mathrm{g} / \mathrm{ml}$ methionine (+Met) and $40 \mu \mathrm{g} / \mathrm{ml}$ uracil (+Ura) was added to media 1-hour prior to imaging. C) Time-lapse microscopy of cells expressing GFP-Snc2 (left) or Fur4-mNG (right). D-E) Wild-type cells expressing fluorescently labelled Snc1, Mup1 and Fur4 were imaged, with example cell cycle stages depicted, and fluorescence in motherdaughter pairs quantified. * denotes $p<0.002$. F-J) Indicated GFP tagged cargoes were imaged by Airyscan microscopy in Sec7-mCherry (upper micrographs) or Vps4-mCherry (lower micrographs) cells, with associated jitter plots of Mander's overlap. * denotes $p<0.0001$ from unpaired $t$-test K) Schematic summarising distinct yeast endosomal recycling pathways.

Scale bar, $5 \mu \mathrm{m}$. 


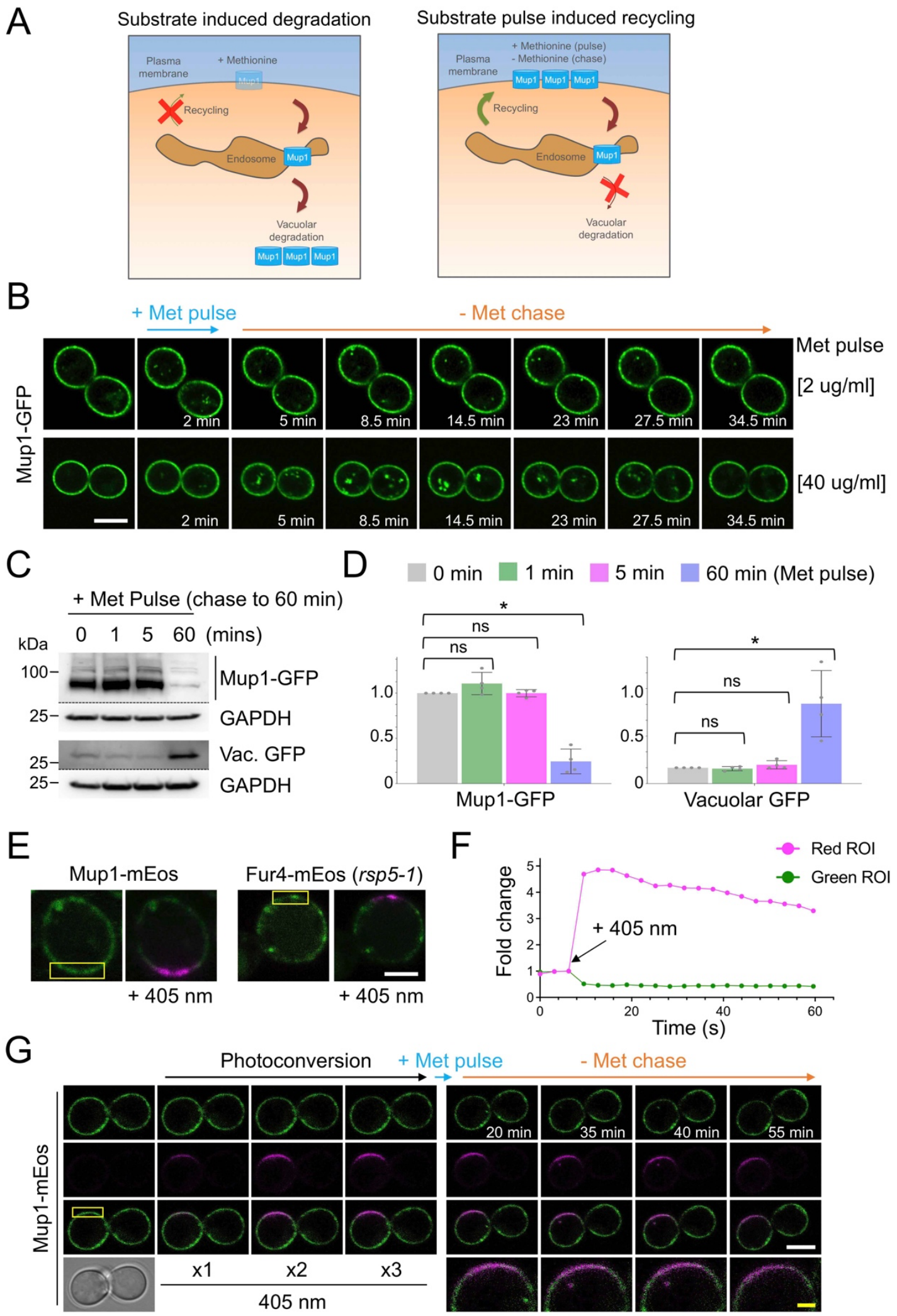

Figure 2: Substrate induced cell surface recycling

A) Cartoon of substrate induced degradation (left) and recycling (right) of Mup1 triggered by modulation of extracellular methionine. B) Time-lapse Airyscan microscopy of cells expressing Mup1-GFP before and after 2-minute methionine $(2 \mu \mathrm{g} / \mathrm{ml}$, upper and $40 \mu \mathrm{g} / \mathrm{ml}$, lower) pulse-chase incubations. C) Cells expressing Mup1-GFP were incubated with $20 \mu \mathrm{g} / \mathrm{ml} \mathrm{methionine}$ for $0,1,5$ and 60 mins followed by $3 x$ washes and further incubation in SC-Met up to 60-minutes before lysates generated and immunobloted. D) Quantification of average intensity of Mup1-GFP (left) and vacuolar processed GFP (right) from methionine pulse-chase experiments from (C). * denotes $p<0.01$. E-F) Yellow regions from cells expressing Mup1-mEos and Fur4-mEos were exposed to $405 \mathrm{~nm}$ laser at $0.5 \%$ to photoconvert molecules (left) and mEOS fluorescence tracked over time before (right). G) Time lapse microscopy of cells expressing Mup1-mEOS following 3x pulse with $0.1 \% 405 \mathrm{~nm}$ laser followed by substrate induced recycling stimulated by $2 \mu \mathrm{g} / \mathrm{ml}$ methionine for 30 seconds. Scale bar represents $5 \mu \mathrm{m}$ (white), $1 \mu \mathrm{m}$ (yellow). 
A

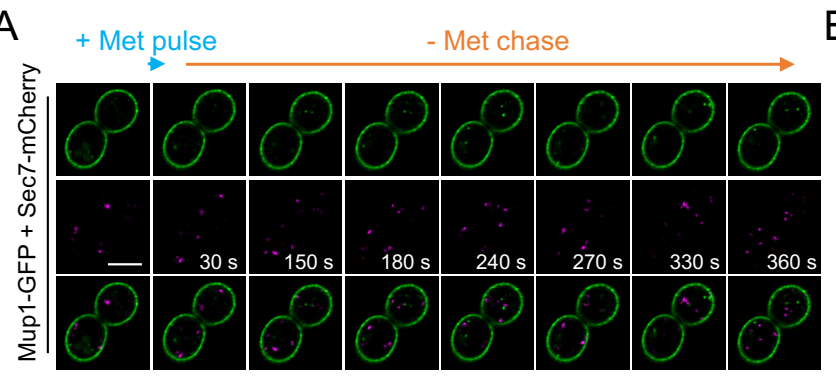

+ Met pulse

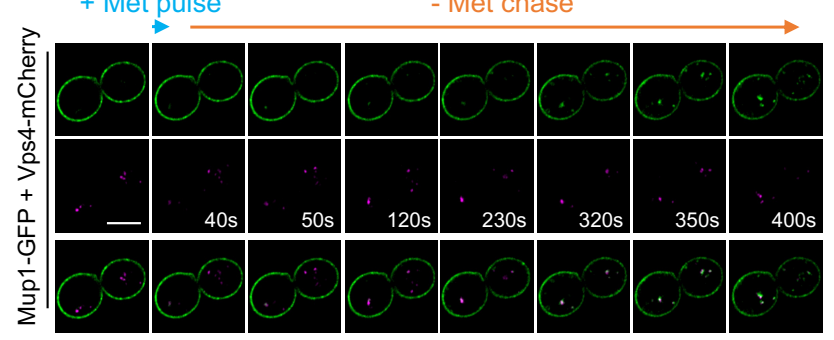

C

C Mup1-GFP + $\bigcirc$ Sec7-mCherry $\bigcirc$ Vps4-mCherry

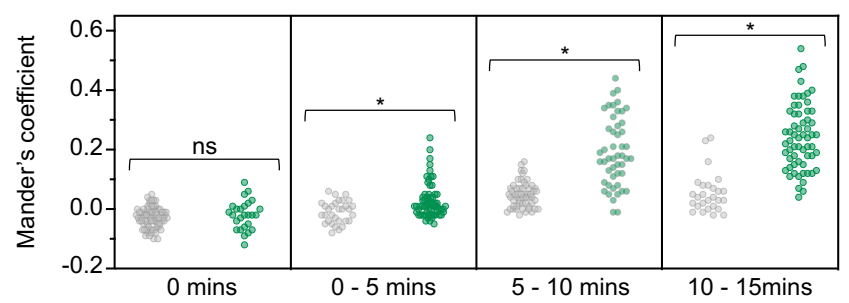

$\mathrm{B}$

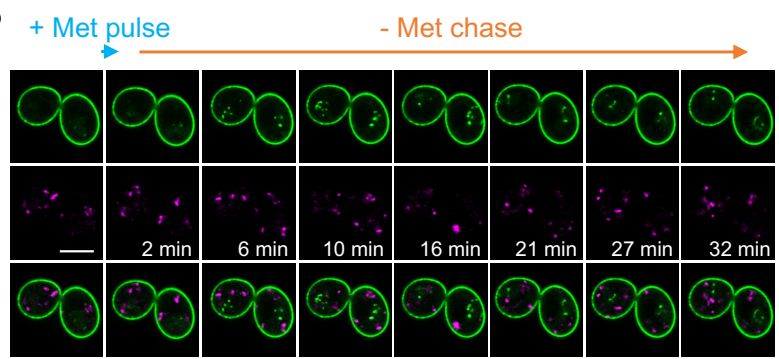

+ Met pulse

- Met chase

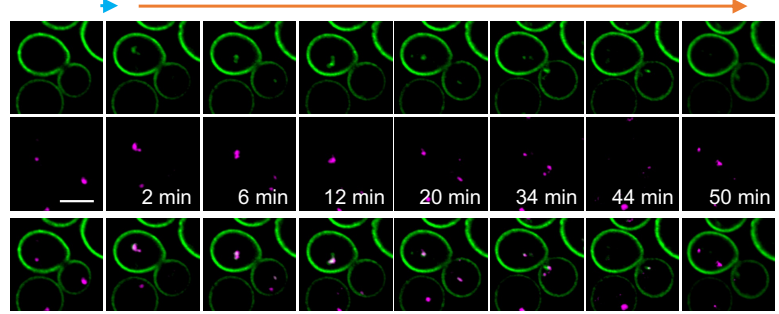

D

Vps4-GFP + Ist1-mCherry

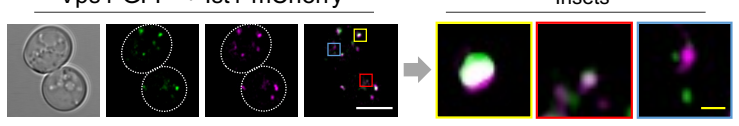

Ist1-GFP + Vps4-mCherry

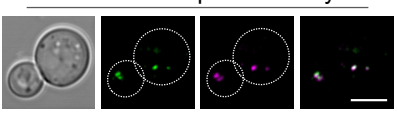

Vps4-mCherry + Sec7-CFP

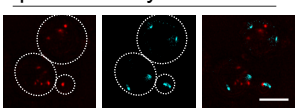

$\mathrm{H}$

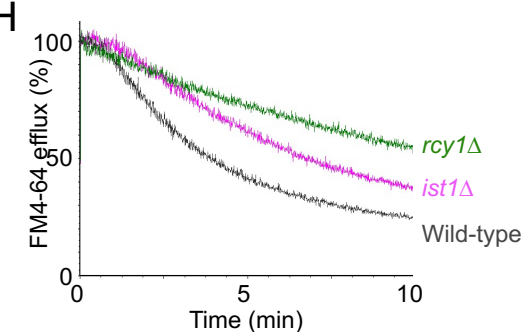

Figure 3: Mup1-GFP recycling occurs from a Vps4-Ist1 endosome

A-C) 4D Airyscan microscopy of wild-type cells co-expressing Mup1-GFP and Sec7-mCherry (upper) and Vps4-mCherry (lower) following a 30 second $20 \mathrm{\mu g} / \mathrm{ml}$ methionine pulse and subsequent SC-Met chase period over short 2-4s (A) and long 30-60s (B) imaging intervals. Quantification of Pearson's correlation coefficients of intercellular Mup1-GFP signal to either Sec7-mCherry (grey) or Vps4-mCherry (green) at indicated times included (C). * denotes $p<0.002$. D) Wild-type cells co-expressing fluorescently labelled versions of Ist1, Vps4 and Sec7 were imaged by Airyscan microscopy. Insets show variation of colocalisation. E) Venn diagram comparing 89 recycling factors with known physical interactors of Vps4. F) Localisation of stably integrated Ste3-GFP-DUb in indicated strains by Airyscan microscopy. G) Histogram showing relative growth of rcy1 $1 \Delta$ and ist $1 \Delta$ mutants compared with wild-type cells across media containing indicated concentrations of tryptophan* represents $p<$ 0.02 from unpaired $t$-test. H) FM4-64 efflux measurements from wild-type, $r c y 1 \Delta$, and ist $1 \Delta$ cells loaded with dye for 8 minutes at room temperature followed by $3 \mathrm{x}$ ice-cold media washes. Scale bars, $5 \mu \mathrm{m}$ (white); $0.5 \mu \mathrm{m}$ (yellow). 
A

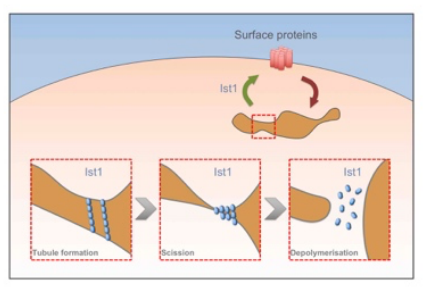

$\mathrm{D}$

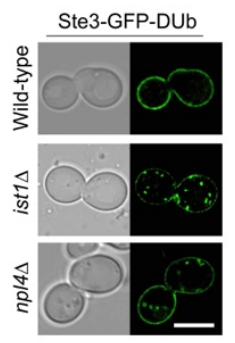

G
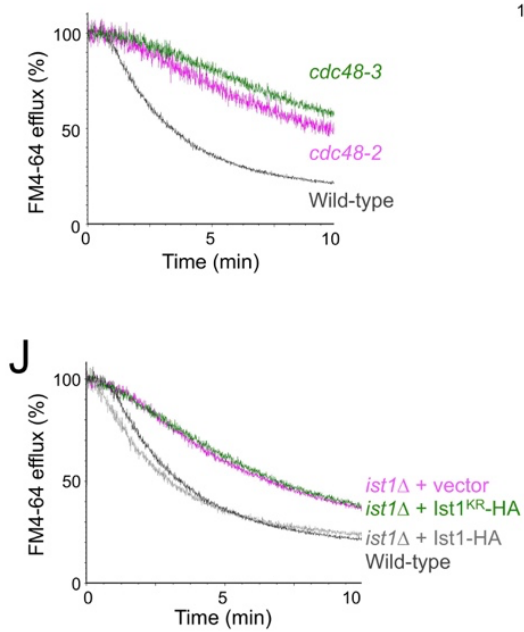

$\mathrm{M}$

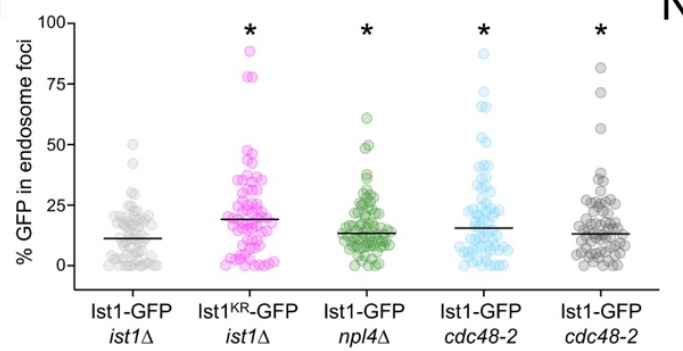

B

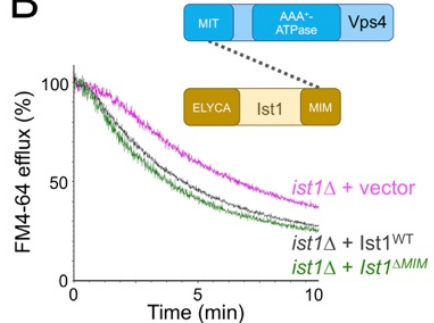

E

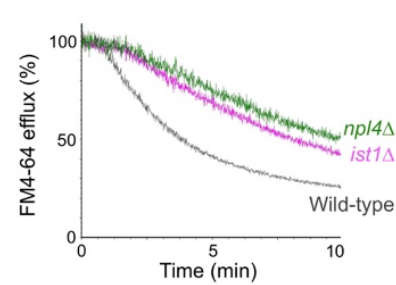

$\mathrm{H}$

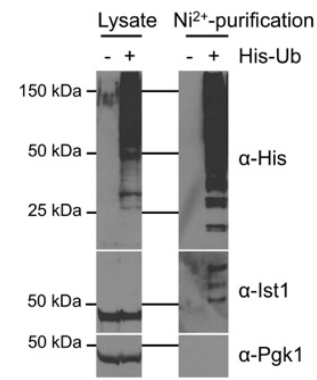

$\mathrm{K}$

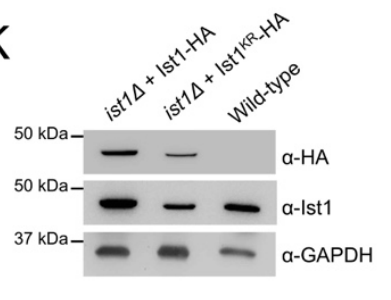

C

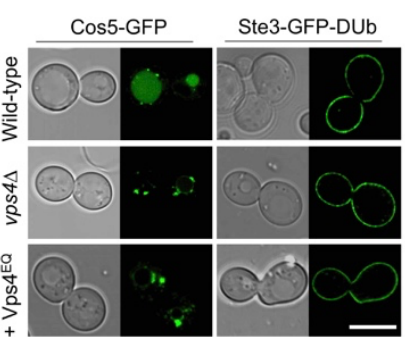

F

cdc48-2

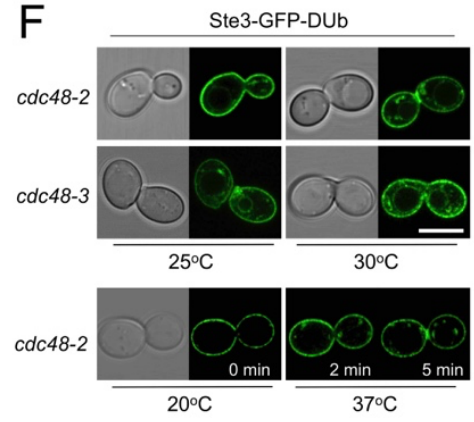

$c d c 48$

cdc48-3

I

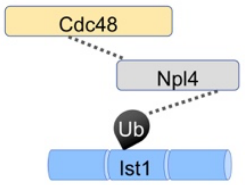

$\mathrm{L}$

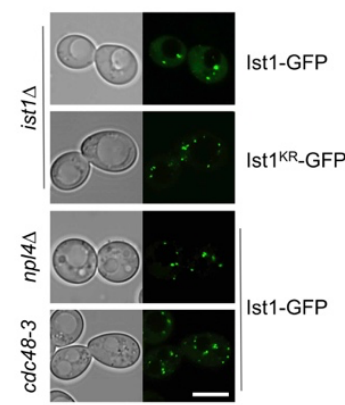

$\mathrm{N}$

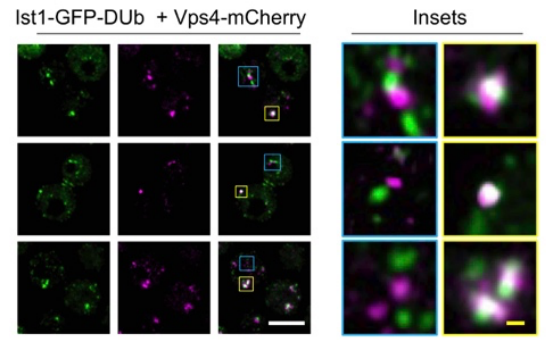

Figure 4: Ubiquitinated Ist1 and Npl4-Cdc48 are required for surface recycling

A) Hypothetical model for the role of Ist1 in recycling. B) FM4-64 efflux measurements following for 8 minutes loading were compared from ist $1 \Delta$ cells transformed with either vector control or plasmids expressing Ist $1^{\mathrm{WT}}$ or Ist $1^{\Delta \mathrm{MIM}}$. Schematic representation of Vps4 and Ist1 interaction (above). C) Airyscan microscopy of MVB cargo (Cos5-GFP) and recycling cargo (Ste3-GFP-DUb) expressed in indicated strains. D) Stably integrated Ste3-GFP-DUb localised in wild-type, ist1 $\Delta$, and $n p / 4 \Delta$ cells by Airyscan microscopy. E) FM4-64 efflux measurements from wild-type, npl4 $\Delta$, and ist1 $\Delta$ cells grown to mid-log phase. F) Stably integrated Ste3-GFP-DUb was expressed in strains harbouring temperature sensitive alleles of CDC48 (cdc48-2 and cdc48-3), grown indicated temperatures then imaged by Airyscan microscopy. G) FM4-64 efflux measurements from wild-type, cdc48-2, and cdc48-3 cells following loading with dye for 8 minutes at room temperature and $3 x$ cold media washes. H) Wildtype and $\mathrm{His}_{6}$-ubiquitin expressing cells were grown to log-phase before ubiquitinated proteins were isolated from a denatured lysate on $\mathrm{Ni}^{2+}$-NTA beads. Original lysates (left) and purified samples (right) were analysed by SDS-PAGE followed by immunoblot with the indicated antibodies. I) Simplified representation of interactions (dotted lines) between Cdc48, Npl4 and Ubiquitin. We hypothesize this enzyme module functionally connects with Ist1 via ubiquitin. J) FM4-64 efflux measurements from wild-type cells and ist $1 \Delta$ cells transformed with plasmids expressing Ist ${ }^{\mathrm{WT}}-\mathrm{HA}$, Ist $1^{\mathrm{KR}}-\mathrm{HA}$, or vector control prior to dye loading for 8 minutes at room temperature. K) Immunoblot of lysates generated from wild-type cells and indicated transformants of ist1 $\Delta$ cells using indicated antibodies. L-M) Indicated strains expressing GFP labelled Ist1 and Ist $1^{\mathrm{KR}}$ were imaged by Airyscan microscopy and \% GFP signal in endosomal foci quantified. N) Airyscan microscopy images of wild-type cells expressing Vps4-mCherry and Ist1-GFP-DUb, including enlarged insets (right). Scale bar, white $5 \mu \mathrm{m}$; yellow $0.5 \mu \mathrm{m}$. 


\section{METHODS}

\section{Reagents}

Supplemental tables are included to document use of plasmids (Table S1), yeast strains (Table S2), primary antibodies (Table S3), and statistical analysis (Table S4).

\section{Cell culture}

Saccharomyces cerevisiae yeast strains were cultured in synthetic complete minimal media (SC; $2 \%$ glucose, yeast nitrogen base supplemented with base / amino acid drop out mixtures for selections) or yeast extract peptone dextrose (YPD; $2 \%$ glucose, $2 \%$ peptone, $1 \%$ yeast extract). Yeast cultures were typically grown in serial dilution overnight to allow for harvesting at mid-log phase $\left(\mathrm{OD}_{600} \leq 1.0\right)$ prior to experimentation, unless otherwise stated. Selection for strains harbouring KanMX cassettes were carried out in rich media containing $250 \mu \mathrm{g} / \mathrm{ml}$ geneticin/G418 (Formedium). The mCherry and GFP fusions of SEC7 were generated with a methotrexate cassette selected on SC media containing $20 \mathrm{mM}$ methotrexate (Alfa Aesar) and $5 \mathrm{mg} / \mathrm{ml}$ sulphanilamide before the loxP flanked cassette was excised by transient expression from a TEF1-Cre plasmid that was subsequently removed via 5-Fluoroorotic Acid media (MacDonald and Piper, 2015) Expression from the CUP1 promoter was induced by the addition of $20-100 \mu \mathrm{M}$ copper chloride. Methionine $(20 \mu \mathrm{g} / \mathrm{ml})$ and uracil $(40 \mu \mathrm{g} / \mathrm{ml})$ were added to SC media to induce trafficking of Mup1 and Fur4, respectively.

\section{Confocal microscopy}

Yeast cells were harvested from early-mid log phase $\left(\mathrm{OD}_{600} \leq 1.0\right)$ and prepared for imaging using Zeiss laser scanning confocal instruments (typically LSM880 equipped with an Airyscan or LSM780/LSM710) using the Plan-Apochromat $63 \times / 1.4$ objective lenses. The fluorescent proteins mCherry, photo-converted mEOS and mStrawberry were excited using the $561 \mathrm{~nm}$ line from a yellow DPSS laser and the emission range 570-620 $\mathrm{nm}$ was collected. The fluorescent proteins mGFP, GFP, mNeonGreen and pre-converted mEOS were excited using the 488nm line from an Argon laser, the emission range 495-550 nm was collected. The fluorescent protein mEOS was photo converted using $0.5 \%$ the $405 \mathrm{~nm}$ laser with 5 iterations per conversion and 3 conversions of a defined region of interested as stated. YPD containing 0.8 $\mu \mathrm{M}$ FM4-64 was used to label vacuoles for 1-hour followed by $3 x$ washing and 1-hour chase period in SC minimal media. For dual population imaging, cultures were grown independently to mid log phase before mixing 1:1 ratio and grown for a further 13 hours. $5 \mu \mathrm{g} / \mathrm{ml} \mathrm{Hoescht} \mathrm{was} \mathrm{added} \mathrm{to} \mathrm{harvested} \mathrm{cells} \mathrm{for} 10$ minutes prior to imaging, and was excited using the $405 \mathrm{~nm}$ line, the emission $460 / 50 \mathrm{~nm}$ was collected.

\section{Microfluidics and time-lapse microscopy}

Yeast cultures were grown to very early log phase $\left(\mathrm{OD}_{600} \leq 0.2\right)$ and adhered to $35 \mathrm{~mm}$ glass bottom coverslip dishes (Ibidi $\mathrm{GmbH}$, Germany) coated with concavalin A (Sigma-Aldrich) prior to live-cell imaging at room temperature. Concavalin A coating was prepared by adding $1 \mathrm{mg} / \mathrm{ml}$ concavalin $A$ in water to the glass bottom coverslip for 5 minutes prior to three washing steps; prepared plates were routinely stored at $4^{\circ} \mathrm{C}$. Sterile media exchanges were performed using $50 \mathrm{~mL}$ syringes through tubing fused to the lid of the $35 \mathrm{~mm}$ dishes.

\section{Image analysis}

Airyscan micrographs were processed using Zen software (Zeiss) and were further modified using Fiji. For timelapse movies, bleach correction was carried out using the inbuilt Fiji plugin and histogram-matching method (Miura, 2020). Any necessary drift correction was carried out in Fiji using the plugins Hyper Stack Reg and Turbo Reg (Thevenaz et al., 1998). Fluorescence intensity measurements during photoconversion experiments was assessed in Zen Black and plotted in GraphPad (v9.0.2, Prism). Steady state colocalization measurements was performed using cell magic wand and morphological erosion to exclude surface signal was used to define intracellular populations that were then analysed by CoLoc2 plugin and Pearson coefficients reported. For timelapse microscopy, Manders overlap coefficients were measured for individual cells after normalisation for individual GFP and mCherry fluorescence using Zen Black (Zeiss).

\section{FM4-64 recycling assay}

Yeast were grown to mid-log phase in SC minimal media with corresponding selection to plasmid or YPD, $1 \mathrm{~mL}$ of cells $(O D=1.0)$ were harvested, incubated for $8 \mathrm{~min}$ at room temperature in $100 \mu \mathrm{L}$ YPD containing $40 \mu \mathrm{M}$ FM4-64 dye (N-(3-Triethylammoniumpropyl)-4-(6-(4-(Diethylamino) Phenyl) Hexatrienyl) Pyridinium Dibromide) dye. Labelled cells were then washed in ice cold SC media for 3 minutes on ice, 3 times. Final wash concentrated cells in $100 \mu \mathrm{L} \mathrm{SC}$ media in preparation for flow cytometry. Approximately 2500 cells were flowed per second at $\sim 600 \mathrm{~V}$ using LSR Fortessa (BD Biosciences), over a 10 min time period the FM4-64 intensity was measured with excitation $561 \mathrm{~nm}$, laser filter $710 / 50$. Background autofluorescence was recorded using measurements from the $530 / 50 \mathrm{~nm}$ detector. 


\section{Immunoblotting}

Equivalent amounts of yeast culture grown to mid log phase $\left(\mathrm{OD}_{600}=<1.0\right)$ were harvested, treated with 500 l $0.2 \mathrm{~N} \mathrm{NaOH}$ for 3 minutes then resuspended in lysis buffer (8 M Urea, $10 \%$ glycerol, $5 \%$ SDS, $10 \% 2$ Mercaptoethanol, $50 \mathrm{mM}$ Tris. $\mathrm{HCl}$ pH 6.8, 0.1\% bromophenol blue). Proteins were resolved using SDS-PAGE and transferred to nitrocellulose membrane using the iBlot2 transfer system (ThermoFisher). The membrane was probed using the labelled antibodies and visualised using super signal Pico Plus (ThermoFisher) Enhanced chemiluminescence signal intensity was captured using an iBright ${ }^{\mathrm{TM}}$ Imager (ThermoFisher).

\section{Statistical tests}

Indicated statistical tests for experimental comparisons were performed using GraphPad (v9.0.2, Prism). An asterisk is used in graphs to denote statistically significant differences $p<0.02$ or less ( $p$ values shown in Table S4).

\section{Bioinformatics}

Gene ontology term finder(Cherry et al., 2012) was used to analyse all the results of the genetic screen for recycling machinery described in(MacDonald and Piper, 2017) with searches for specific enzyme activity shown. The physical interactome was acquired from YeastMine (Balakrishnan et al., 2012)

\section{Tat2 recycling assay}

Tryptophan auxotroph (trp1 $\Delta$ ) yeast cells were grown to mid log-phase before being spotted out across a 10 fold serial dilution and grown on plates of indicated Tryptophan concentrations. Yeast growth was normalised from a wild-type control on the same plate and used the calculate the growth difference, as previously described (Paine et al., 2021).

\section{Ubiquitome purification}

Yeast CMY158 optimised for ubiquitin purification (MacDonald et al., 2017, 2020) expressing His6-tagged ubiquitin were grown over night and used to inoculate a 1 litre culture that was grown to mid-log phase before cells were harvested, treated with $0.2 \mathrm{~N}$ sodium hydroxide and brought up in denaturing lysis buffer (8 $\mathrm{M}$ urea, $50 \mathrm{mM}$ Tris. $\mathrm{HCl} \mathrm{pH} \mathrm{8.5,5 \%} \mathrm{(w/v)} \mathrm{glycerol,} \mathrm{2.5 \%} \mathrm{SDS,} 5 \mathrm{mM}$ 2-mercaptoethanol). Lysates were diluted in lysis buffer lacking SDS and used to bind $2 \mathrm{ml}$ bed of $\mathrm{Ni}^{2+} \mathrm{NTA}$ agarose for 2-hours. Beads were incubated 5 times with wash buffer $(50 \mathrm{mM}$ Tris. $\mathrm{HCl} \mathrm{pH} \mathrm{8.5,5 \%} \mathrm{(w/v)} \mathrm{glycerol,} 8 \mathrm{M}$ urea, $5 \mathrm{mM}$ 2-mercaptoethanol) containing 10 $\mathrm{mM}$ imidazole before elution in wash buffer at $\mathrm{pH}$ 4.5. Sample was then neutralised, bound to $200 \mu \mathrm{l}$ bed of $\mathrm{Ni}^{2+-}$ NTA agarose for a further 2 hours, before repeat washes and elutions performed in wash buffer containing 350 $\mathrm{mM}$ imidazole. Loading buffer was added to samples and downstream SDS-PAGE and immunoblot analysis.

\section{ACKNOWLEDGMENTS}

We would like to thank Dave Katzmann (Mayo Clinic) for Ist1 antibodies and expression plasmids; David Teis (University of Innsbruck) and Jeff Brodsky (University of Pittsburgh) for yeast strains; Rob Piper (University of lowa), Markus Babst (University of Utah), and Chris Stefan (LMCB, UCL) for helpful discussions. Thanks also to staff at York Bioscience Technology Facility, in particular Pete O'Toole, Jo Marrison, Karen Hogg and Graeme Park for help with imaging and photoconversion. Finally, thanks to Sarah Lecinski for advice on data analysis and Katherine Paine for comments on the manuscript. This research was supported by a Sir Henry Dale Research Fellowship from the Wellcome Trust and the Royal Society 204636/Z/16/Z (CM).

\section{DECLARATION OF INTERESTS}

The authors declare no competing interests. 


\section{REFERENCES}

Adell, M.A.Y., Migliano, S.M., Upadhyayula, S., Bykov, Y.S., Sprenger, S., Pakdel, M., Vogel, G.F., Jih, G., Skillern, W., Behrouzi, R., et al. (2017). Recruitment dynamics of ESCRT-III and Vps4 to endosomes and implications for reverse membrane budding. Elife 6, e31652.

Allison, R., Lumb, J.H., Fassier, C., Connell, J.W., Martin, D.T., Seaman, M.N.J., Hazan, J., and Reid, E. (2013). An ESCRT-spastin interaction promotes fission of recycling tubules from the endosome. J Cell Biology 202, 527543.

Allison, R., Edgar, J.R., Pearson, G., Rizo, T., Newton, T., Günther, S., Berner, F., Hague, J., Connell, J.W., Winkler, J., et al. (2017). Defects in ER-endosome contacts impact lysosome function in hereditary spastic paraplegia. J Cell Biol $216,13371355$.

Azmi, I., Davies, B., Dimaano, C., Payne, J., Eckert, D., Babst, M., and Katzmann, D.J. (2006). Recycling of ESCRTs by the AAA-ATPase Vps4 is regulated by a conserved VSL region in Vta1. J Cell Biology 172, 705717.

Babst, M., Sato, T.K., Banta, L.M., and Emr, S.D. (1997). Endosomal transport function in yeast requires a novel AAAtype ATPase, Vps4p. Embo J 16, 18201831.

Babst, M., Wendland, B., Estepa, E.J., and Emr, S.D. (1998). The Vps4p AAA ATPase regulates membrane association of a Vps protein complex required for normal endosome function. Embo J 17, 29822993.

Babst, M., Katzmann, D.J., Estepa-Sabal, E.J., Meerloo, T., and Emr, S.D. (2002). Escrt-III: an endosome-associated heterooligomeric protein complex required for mvb sorting. Developmental Cell 3, 271282.

Balakrishnan, R., Park, J., Karra, K., Hitz, B.C., Binkley, G., Hong, E.L., Sullivan, J., Micklem, G., and Cherry, J.M. (2012). YeastMine - an integrated data warehouse for Saccharomyces cerevisiae data as a multipurpose tool-kit.

Database 2012, bar062.

Best, J.T., Xu, P., McGuire, J.G., Leahy, S.N., and Graham, T.R. (2020). Yeast synaptobrevin, Snc1, engages distinct routes of postendocytic recycling mediated by a sorting nexin, Rcy1-COPI, and retromer. Mol Biol Cell 31, 944-962. Buono, R.A., Paez-Valencia, J., Miller, N.D., Goodman, K., Spitzer, C., Spalding, E.P., and Otegui, M.S. (2016). Role of SKD1 Regulators LIP5 and IST1-LIKE1 in Endosomal Sorting and Plant Development. Plant Physiol 171, 251-264. Chen, S.H., Chen, S., Tokarev, A.A., Liu, F., Jedd, G., and Segev, N. (2005). Ypt31/32 GTPases and their novel F-box effector protein Rcyl regulate protein recycling. Mol Biol Cell 16, 178192.

Cherry, J.M., Hong, E.L., Amundsen, C., Balakrishnan, R., Binkley, G., Chan, E.T., Christie, K.R., Costanzo, M.C., Dwight, S.S., Engel, S.R., et al. (2012). Saccharomyces Genome Database: the genomics resource of budding yeast. Nucleic Acids Res 40, D700-D705.

Crespo-Yàñez, X., Aguilar-Gurrieri, C., Jacomin, A.-C., Journet, A., Mortier, M., Taillebourg, E., Soleilhac, E., Weissenhorn, W., and Fauvarque, M.-O. (2018). CHMP1B is a target of USP8/UBPY regulated by ubiquitin during endocytosis. Plos Genet 14, e1007456.

Davis, N., Horecka, J., and Sprague, G. (1993). Cis- and trans-acting functions required for endocytosis of the yeast pheromone receptors. J Cell Biology 122, 53-65.

Day, K.J., Casler, J.C., and Glick, B.S. (2018). Budding Yeast Has a Minimal Endomembrane System. Dev Cell 44, 56 72.e4.

Dimaano, C., Jones, C.B., Hanono, A., Curtiss, M., and Babst, M. (2008). Ist1 regulates Vps4 localization and assembly. Mol Biol Cell 19, 465474.

Frankel, E.B., Shankar, R., Moresco, J.J., Yates, J.R., Volkmann, N., and Audhya, A. (2017). Ist1 regulates ESCRT-III assembly and function during multivesicular endosome biogenesis in Caenorhabditis elegans embryos. Nat Commun 8 , 1439.

Furuta, N., Fujimura-Kamada, K., Saito, K., Yamamoto, T., and Tanaka, K. (2007). Endocytic recycling in yeast is regulated by putative phospholipid translocases and the Ypt31p/32p-Rcylp pathway. Mol Biol Cell 18, 295312.

Galan, J.-M., Wiederkehr, A., Seol, J.H., Haguenauer-Tsapis, R., Deshaies, R.J., Riezman, H., and Peter, M. (2001). Skplp and the F-Box Protein Rcylp Form a Non-SCF Complex Involved in Recycling of the SNARE Snc1p in Yeast. Mol Cell Biol 21, 31053117.

Goldenring, J.R. (2015). Recycling endosomes. Curr Opin Cell Biol 35, 117-122.

Grant, B.D., and Donaldson, J.G. (2009). Pathways and mechanisms of endocytic recycling. Nat Rev Mol Cell Bio 10, 597608.

Han, H., Monroe, N., Sundquist, W.I., Shen, P.S., and Hill, C.P. (2017). The AAA ATPase Vps4 binds ESCRT-III substrates through a repeating array of dipeptide-binding pockets. Elife 6, e31324.

Hanamatsu, H., Fujimura-Kamada, K., Yamamoto, T., Furuta, N., and Tanaka, K. (2014). Interaction of the phospholipid flippase Drs2p with the F-box protein Rcy 1p plays an important role in early endosome to trans-Golgi network vesicle transport in yeast. J Biochem 155, 5162.

Hanson, P.I., Roth, R., Lin, Y., and Heuser, J.E. (2008). Plasma membrane deformation by circular arrays of ESCRT-III protein filaments. J Cell Biology 180, 389402.

Hein, C., Springael, J., Volland, C., Haguenauer-Tsapis, R., and André, B. (1995). NPI1, an essential yeast gene involved in induced degradation of Gap1 and Fur4 permeases, encodes the Rsp5 ubiquitin — protein ligase. Mol Microbiol 18, 77-87. 
Hettema, E.H., Lewis, M.J., Black, M.W., and Pelham, H.R.B. (2003). Retromer and the sorting nexins Snx4/41/42 mediate distinct retrieval pathways from yeast endosomes. Embo J 22, 548557.

Hua, Z., Fatheddin, P., and Graham, T.R. (2002). An essential subfamily of Drs2p-related P-type ATPases is required for protein trafficking between Golgi complex and endosomal/vacuolar system. Mol Biol Cell 13, 31623177.

Isnard, A.-D., Thomas, D., and Surdin-Kerjan, Y. (1996). The Study of Methionine Uptake inSaccharomyces cerevisiaeReveals a New Family of Amino Acid Permeases. J Mol Biol 262, 473-484.

Johnson, S.S., Hanson, P.K., Manoharlal, R., Brice, S.E., Cowart, L.A., and Moye-Rowley, W.S. (2010). Regulation of yeast nutrient permease endocytosis by ATP-binding cassette transporters and a seven-transmembrane protein, RSB1. J Biol Chem 285, 3579235802.

Jones, C.B., Ott, E.M., Keener, J.M., Curtiss, M., Sandrin, V., and Babst, M. (2012). Regulation of Membrane Protein Degradation by Starvation-Response Pathways. Traffic 13, 468482.

Katzmann, D.J., Babst, M., and Emr, S.D. (2001). Ubiquitin-Dependent Sorting into the Multivesicular Body Pathway Requires the Function of a Conserved Endosomal Protein Sorting Complex, ESCRT-I. Cell 106, 145155.

Keener, J.M., and Babst, M. (2013). Quality Control and Substrate-Dependent Downregulation of the Nutrient Transporter Fur4. Traffic 14, 412427.

Laidlaw, K.M.E., and MacDonald, C. (2018). Endosomal trafficking of yeast membrane proteins. Biochem Soc T 46, $1551-1558$.

Laidlaw, K.M.E., Bisinski, D.D., Shashkova, S., Paine, K.M., Veillon, M.A., Leake, M.C., and MacDonald, C. (2020). A glucose-starvation response governs endocytic trafficking and eisosomal retention of surface cargoes in budding yeast. $\mathrm{J}$ Cell Sci 134, jcs257733.

Lewis, M.J., Nichols, B.J., Prescianotto-Baschong, C., Riezman, H., and Pelham, H.R. (2000). Specific retrieval of the exocytic SNARE Snclp from early yeast endosomes. Molecular Biology of the Cell 11, 2338.

Lin, C.H., MacGurn, J.A., Chu, T., Stefan, C.J., and Emr, S.D. (2008). Arrestin-Related Ubiquitin-Ligase Adaptors Regulate Endocytosis and Protein Turnover at the Cell Surface. Cell 135, 714725.

Ma, M., and Burd, C.G. (2019). Retrograde trafficking and plasma membrane recycling pathways of the budding yeast Saccharomyces cerevisiae. Traffic.

Ma, M., Burd, C.G., and Chi, R.J. (2017). Distinct complexes of yeast Snx4 family SNX-BARs mediate retrograde trafficking of Snc1 and Atg27. Traffic 18, 134144.

MacDonald, C., and Piper, R.C. (2015). Puromycin- and methotrexate-resistance cassettes and optimized Crerecombinase expression plasmids for use in yeast. Yeast 32, 423438.

MacDonald, C., and Piper, R.C. (2016). Cell surface recycling in yeast: mechanisms and machineries. Biochem Soc T 44, 474478.

MacDonald, C., and Piper, R.C. (2017). Genetic dissection of early endosomal recycling highlights a TORC1independent role for Rag GTPases. J Cell Biol 8, jcb.201702177.

MacDonald, C., Buchkovich, N.J., Stringer, D.K., Emr, S.D., and Piper, R.C. (2012a). Cargo ubiquitination is essential for multivesicular body intralumenal vesicle formation. Embo Rep 13, 331338.

MacDonald, C., Stringer, D.K., and Piper, R.C. (2012b). Sna3 Is an Rsp5 Adaptor Protein that Relies on Ubiquitination for Its MVB Sorting. Traffic 13, 586598.

MacDonald, C., Payne, J.A., Aboian, M., Smith, W., Katzmann, D.J., and Piper, R.C. (2015). A Family of Tetraspans Organizes Cargo for Sorting into Multivesicular Bodies. Dev Cell 33, 328342.

MacDonald, C., Winistorfer, S., Pope, R.M., Wright, M.E., and Piper, R.C. (2017). Enzyme reversal to explore the function of yeast E3 ubiquitin-ligases. Traffic 18, 465484.

MacDonald, C., Shields, S.B., Williams, C.A., Winistorfer, S., and Piper, R.C. (2020). A Cycle of Ubiquitination Regulates Adaptor Function of the Nedd4-Family Ubiquitin Ligase Rsp5. Curr Biology Cb.

MacGurn, J.A., Hsu, P.-C., Smolka, M.B., and Emr, S.D. (2011). TORC1 Regulates Endocytosis via Npr1-Mediated Phosphoinhibition of a Ubiquitin Ligase Adaptor. Cell 147, 11041117.

Maity, S., Caillat, C., Miguet, N., Sulbaran, G., Effantin, G., Schoehn, G., Roos, W.H., and Weissenhorn, W. (2019). VPS4 triggers constriction and cleavage of ESCRT-III helical filaments. Sci Adv 5, eaau7198.

McCullough, J., Clippinger, A.K., Talledge, N., Skowyra, M.L., Saunders, M.G., Naismith, T.V., Colf, L.A., Afonine, P., Arthur, C., Sundquist, W.I., et al. (2015). Structure and membrane remodeling activity of ESCRT-III helical polymers. Science 350, 15481551.

Menant, A., Barbey, R., and Thomas, D. (2006). Substrate-mediated remodeling of methionine transport by multiple ubiquitin-dependent mechanisms in yeast cells. Embo J 25, 4436-4447.

Miura, K. (2020). Bleach correction ImageJ plugin for compensating the photobleaching of time-lapse sequences. F1000research 9, 1494.

Müller, M., Schmidt, O., Angelova, M., Faserl, K., Weys, S., Kremser, L., Pfaffenwimmer, T., Dalik, T., Kraft, C., Trajanoski, Z., et al. (2015). The coordinated action of the MVB pathway and autophagy ensures cell survival during starvation. Elife 4, e07736. 
Nguyen, H.C., Talledge, N., McCullough, J., Sharma, A., Moss, F.R., Iwasa, J.H., Vershinin, M.D., Sundquist, W.I., and Frost, A. (2020). Membrane constriction and thinning by sequential ESCRT-III polymerization. Nat Struct Mol Biol 27, 392-399.

Nickerson, D.P., West, M., and Odorizzi, G. (2006). Did2 coordinates Vps4-mediated dissociation of ESCRT-III from endosomes. J Cell Biology 175, 715720.

O'Sullivan, M.J., and Lindsay, A.J. (2020). The Endosomal Recycling Pathway-At the Crossroads of the Cell. Int J Mol Sci 21, 6074.

Paine, K.M., Ecclestone, G.B., and MacDonald, C. (2021). Fur4 mediated uracil-scavenging to screen for surface protein regulators. Biorxiv 2021.05.27.445995.

Pfitzner, A.-K., Mercier, V., Jiang, X., Filseck, J.M. von, Baum, B., Šarić, A., and Roux, A. (2020). An ESCRT-III Polymerization Sequence Drives Membrane Deformation and Fission. Cell 182, 1140-1155.e18.

Protopopov, V., Govindan, B., Novick, P., and Gerst, J.E. (1993). Homologs of the synaptobrevin/VAMP family of synaptic vesicle proteins function on the late secretory pathway in S. cerevisiae. Cell 74, 855-861.

Rue, S.M., Mattei, S., Saksena, S., and Emr, S.D. (2008). Novel Ist1-Did2 complex functions at a late step in multivesicular body sorting. Mol Biol Cell 19, 475484.

Saksena, S., Wahlman, J., Teis, D., Johnson, A.E., and Emr, S.D. (2009). Functional Reconstitution of ESCRT-III Assembly and Disassembly. Cell 136, 97109.

Sardana, R., and Emr, S.D. (2021). Membrane Protein Quality Control Mechanisms in the Endo-Lysosome System. Trends Cell Biol.

Schothorst, J., Kankipati, H.N., Conrad, M., Samyn, D.R., Zeebroeck, G.V., Popova, Y., Rubio-Texeira, M., Persson, B.L., and Thevelein, J.M. (2013). Yeast nutrient transceptors provide novel insight in the functionality of membrane transporters. Curr Genet 59, 197-206.

Seaman, M.N., McCaffery, J.M., and Emr, S.D. (1998). A membrane coat complex essential for endosome-to-Golgi retrograde transport in yeast. The Journal of Cell Biology 142, 665681.

Séron, K., Blondel, M.-O., Haguenauer-Tsapis, R., and Volland, C. (1999). Uracil-Induced Down-Regulation of the Yeast Uracil Permease. J Bacteriol 181, 1793-1800.

Shestakova, A., Hanono, A., Drosner, S., Curtiss, M., Davies, B.A., Katzmann, D.J., and Babst, M. (2010). Assembly of the AAA ATPase Vps4 on ESCRT-III. Mol Biol Cell 21, 10591071.

Shi, Y., Stefan, C.J., Rue, S.M., Teis, D., and Emr, S.D. (2011). Two novel WD40 domain-containing proteins, Ere1 and Ere2, function in the retromer-mediated endosomal recycling pathway. Mol Biol Cell 22, 40934107.

Shields, S.B., Oestreich, A.J., Winistorfer, S., Nguyen, D., Payne, J.A., Katzmann, D.J., and Piper, R. (2009). ESCRT ubiquitin-binding domains function cooperatively during MVB cargo sorting. J Cell Biology 185, 213224.

Stringer, D.K., and Piper, R.C. (2011). A single ubiquitin is sufficient for cargo protein entry into MVBs in the absence of ESCRT ubiquitination. J Cell Biology 192, 229242.

Tan, J., Davies, B.A., Payne, J.A., Benson, L.M., and Katzmann, D.J. (2015). Conformational Changes in the Endosomal Sorting Complex Required for the Transport III Subunit Ist1 Lead to Distinct Modes of ATPase Vps4 Regulation. J Biol Chem 290, 3005330065.

Tang, S., Henne, W.M., Borbat, P.P., Buchkovich, N.J., Freed, J.H., Mao, Y., Fromme, J.C., and Emr, S.D. (2015).

Structural basis for activation, assembly and membrane binding of ESCRT-III Snf7 filaments. Elife 4, e12548.

Teis, D., Saksena, S., and Emr, S.D. (2008). Ordered Assembly of the ESCRT-III Complex on Endosomes Is Required to Sequester Cargo during MVB Formation. Dev Cell 15, 578589.

Thevenaz, P., Ruttimann, U.E., and Unser, M. (1998). A pyramid approach to subpixel registration based on intensity. Ieee T Image Process 7, 27-41.

Urbanowski, J.L., and Piper, R.C. (2001). Ubiquitin Sorts Proteins into the Intralumenal Degradative Compartment of the Late-Endosome/Vacuole. Traffic 2, 622630.

Valdez-Taubas, J., and Pelham, H.R.B. (2003). Slow Diffusion of Proteins in the Yeast Plasma Membrane Allows Polarity to Be Maintained by Endocytic Cycling. Curr Biol 13, 1636-1640.

Wang, B., Alam, S.L., Meyer, H.H., Payne, M., Stemmler, T.L., Davis, D.R., and Sundquist, W.I. (2003). Structure and Ubiquitin Interactions of the Conserved Zinc Finger Domain of Npl4*. J Biol Chem 278, 20225-20234.

Wiederkehr, A., Avaro, S., Prescianotto-Baschong, C., Haguenauer-Tsapis, R., and Riezman, H. (2000). The F-Box Protein Rcy1p Is Involved in Endocytic Membrane Traffic and Recycling Out of an Early Endosome in Saccharomyces cerevisiae. J Cell Biology 149, 397-410.

Wollert, T., Wunder, C., Lippincott-Schwartz, J., and Hurley, J.H. (2009). Membrane scission by the ESCRT-III complex. Nature 458, 172177.

Xiao, J., Chen, X.-W., Davies, B.A., Saltiel, A.R., Katzmann, D.J., and Xu, Z. (2009). Structural basis of Ist1 function and Ist1-Did2 interaction in the multivesicular body pathway and cytokinesis. Mol Biol Cell 20, 35143524.

Xu, P., Hankins, H.M., MacDonald, C., Erlinger, S.J., Frazier, M.N., Diab, N.S., Piper, R.C., Jackson, L.P., MacGurn, J.A., and Graham, T.R. (2017). COPI mediates recycling of an exocytic SNARE by recognition of a ubiquitin sorting signal. Elife 6, e28342. 